\title{
BURNOUT NO ESPORTE: REVISAO SISTEMATICA NA BASE ScienceDirect
}

\author{
Alexandro Andrade* \\ Pedro de Orleans Casagrande ** \\ Ricardo Brandt *** \\ Maick da Silveira Viana ${ }^{* \star * *}$
}

\begin{abstract}
RESUMO
O presente estudo teve por objetivo analisar a produção científica relacionada ao burnout nos esportes a partir de artigos publicados na última década (2002 a 2011) na base de dados eletrônica ScienceDirect, por ser esta relevante, reconhecida, representativa e abrangente. A partir das combinações de palavras "burnout e sports" e "burnout e athletes", oito artigos foram selecionados para a pesquisa. Os itens investigados foram:revista, tipo de estudo, número de participantes, amostra, população investigada,instrumento utilizados, tema e resultados. As publicações se concentraram nas revistas Journal of Science and Medicine in Sport e Psychology of Sport and Exercise, predominaram pesquisas quantitativas $(n=7)$ com uma média de 228 participantes. As populações investigadas foram de atletas de elite juvenis, atletas quase profissionais e atletas profissionais de diferentes países, europeus e da Oceania. Todos os estudos utilizaram o Athlete Burnout Questionaire (ABQ) como instrumento de coleta de dados. Quanto ao tema, foram identificados e apresentados os resultados de artigos sobre burnout e perfeccionismo $(n=2)$, sinais que antecedem o burnout $(n=2)$, burnout, seus antecedentes e conseqüências para atletas que abandonaram o esporte $(n=1)$, variações nos níveis de burnout ao longo de uma temporada competitiva $(n=1)$, paixão pelo esporte e burnout $(n=1)$ e clima motivacional e o burnout $(n=1)$.
\end{abstract}

Palavras-chave:Burnout. Esporte. Atleta. Psicologia.

\section{INTRODUÇÃO}

O burnout tem sido tema de estudo de autores de diferentes áreas da Psicologia, sendo que Freudenberger foi um de seus pioneiros. Freudenberger (1974) definiu o burnout como um sentimento de fracasso, esgotamento e exaustão diante de excessivas demandas energéticas. Maslack e Jackson (1981), a partir de estudos realizados em organizações, conceituaram o burnout como uma síndrome tridimensional que atingia os trabalhadores, caracterizada por esgotamento emocional, despersonalização e reduzida realização pessoal. As mesmas autoras ampliaram as investigações a outros âmbitos da atividade humana, incluindo o esporte, o qual recebeu investigações específicas a partir dos trabalhos de Flippin (1981).

Raedeke's (1997), baseado nos estudos de Maslack e Jackson (1981), adaptou as dimensões da teoria que eram direcionadas para as organizações

\footnotetext{
* Prof. Dr. do Programa de Pós-Graduação em Ciências do Movimento Humano da Universidade do Estado de Santa Catarina UDESC. Coordenador do Laboratório de Psicologia do Esporte e do Exercício - LAPE.

** Bacharel em Educação Física. Pesquisador do Laboratório de Psicologia do Esporte e do Exercício - LAPE.

*** Licenciado em Educação Física. Doutorando em Ciências do Movimento Humano pela Universidade do Estado de Santa Catarina - UDESC. Pesquisador do Laboratório de Psicologia do Esporte e do Exercício - LAPE.

****Licenciado em Educação Física - UDESC Mestre em Ciências do Movimento Humano - UDESC Doutorando em Ciênias do Movimento Humano - UDESC Pesquisador do Laboratório de Psicologia do Esporte e do Exercício - LAPE.
} 
ao contexto esportivo. O resultado foi a definição do burnout esportivo como uma síndrome psicológica fundamentada em três dimensões: "exaustão física e emocional", "desvalorização esportiva" e "baixo senso de realização esportiva" (GUSTAFSSON et al., 2008; CRESSWELL; EKLUND, 2006; RAEDEKE; LUNNEY; VENABLES, 2002).

A "exaustão física e emocional" está associada aos treinamentos intensos e às competições. $O$ atleta passa a sentir-se cansado e exausto com frequência. Em relação à "desvalorização esportiva", o atleta passa a sentir aversão pelo treinamento e competições, tendo atitudes negativas em relação ao esporte e desvalorizando-o em comparação a outras atividades de sua vida. A dimensão "baixo senso de realização esportiva" está relacionada ao desempenho das habilidades, sendo que o atleta pode se frustrar por não atingir suas metas pessoais ou seu rendimento estar abaixo de suas expectativas (GUSTAFSSON et al., 2008; GOODGER et al., 2007).

Por se tratar de um conjunto de sintomas de ordem física e psicológica, segundo Pires, Brandão e Machado (2005), muitas vezes o burnout não é identificado em curto prazo entre os atores do meio esportivo, como atletas, treinadores, árbitros e até mesmo os dirigentes esportivos. Por outro lado, o burnout é relevante para pesquisadores, treinadores e atletas por ter muitas vezes como conseqüência o abandono da prática esportiva (GARCE DE LOS FAYOS; VALLERINO, 2010). É importante considerar ainda que, mesmo quando não há o abandono, o burnout pode ser motivo de sofrimento psíquico para o indivíduo que convive com ele (RAEDEKE, 1997; SMITH, 1986).

Para Pires, Brandão e Machado (2005), apesar de sua relevância, o burnout ainda tem recebido pouca atenção na área esportiva. Visando contribuir com a sistematização do conhecimento produzido na área, o objetivo da presente pesquisa foi analisar a produção científica relacionada ao burnout nos esportes a partir de artigos publicados na última década (2002 a 2011). Uma discussão em torno da produção científica sobre burnout no esporte pode ser útil para o desenvolvimento do esporte, em especial apoiando técnicos e atletas, e da Psicologia do Esporte enquanto área científica. 


\section{MÉTODO}

Trata-se de uma revisão sistemática da literatura, na qual os dados utilizados para a investigação são provenientes de estudos selecionados por meio de um método sistemático descrito a seguir.

\section{Procedimentos da pesquisa}

Este estudo delimitou-se a investigar a produção científica sobre burnout esportivo publicada em periódicos indexados na base de dados eletrônica ScienceDirect. A busca pelos artigos foi realizada no mês de fevereiro de 2012. Analisou-se a produção científica relacionada ao burnout nos esportes a partir de artigos publicados na última década (2002 a 2011) no ScienceDirect, por ser esta relevante, reconhecida, representativa e abrangente. O ScienceDirect atende aos critérios de abrangência e representatividade, por indexar grande parte dos mais importantes periódicos científicos relacionados ao esporte, e de acessibilidade, por dispor seus artigos na integra.. A partir das combinações de palavras "burnout e sports" e "burnout e athletes", oito artigos foram selecionados para a pesquisa. Os itens investigados foram:revista, tipo de estudo, número de participantes, amostra, população investigada,instrumento utilizados, tema e resultados.

Trata-se de um banco de dados que disponibiliza produções publicadas em mais de 2.500periódicos e 11.000livros.Atualmente podem ser acessados de 9,5milhões de artigose capítulos de livros, com crescimento dequase 0,5milhões de adiçõespor ano. Além disso, oScienceDirectfaz parte daElsevier, que tem sede em Amsterdã, na Holanda. A Elsevier é fornecedoramundial deinformação científica,técnica e médicae publicamais de 2.000revistas, bem como livros e bases de dadossecundárias (SCIENCE DIRECT). O aparecimento dos periódicos eletrônicos foi uma grande mudança que ocorreu na década de 1990, e atualmente é um dos meios mais usados para a difusão do conhecimento (FERREIRA NETO; NASCIMENTO, 2002). Tais publicações constituem um importante pólo de divulgação científica, principalmente devido à possibilidade de permanência dos registros e seu alcance geográfico.

As pesquisas encontradas e analisadas neste estudo foram publicadas nas revistas Journal of Science and Medicine in Sport e Psychology of Sport 
and Exercise. O Journal of Sports Science and Medicinein Sport é uma revista científica que publica artigos nos campos da Medicina Esportiva e Ciências do Exercício (JSSM), enquanto o Psychology of Sport and Exercise tem como foco publicar artigos na área da Psicologia do Esporte e do Exercício (PSE).

Utilizou-se para a pesquisa o termo burnout associado aos termos athletes ou sports. Foram utilizados como filtro para a pesquisa os termos encontrados no resumo, título e palavras-chave. Como abrangência da busca, delimitou-se os artigos publicados no período compreendido entre os anos de 2002 e 2011, contemplando os últimos 10 anos.

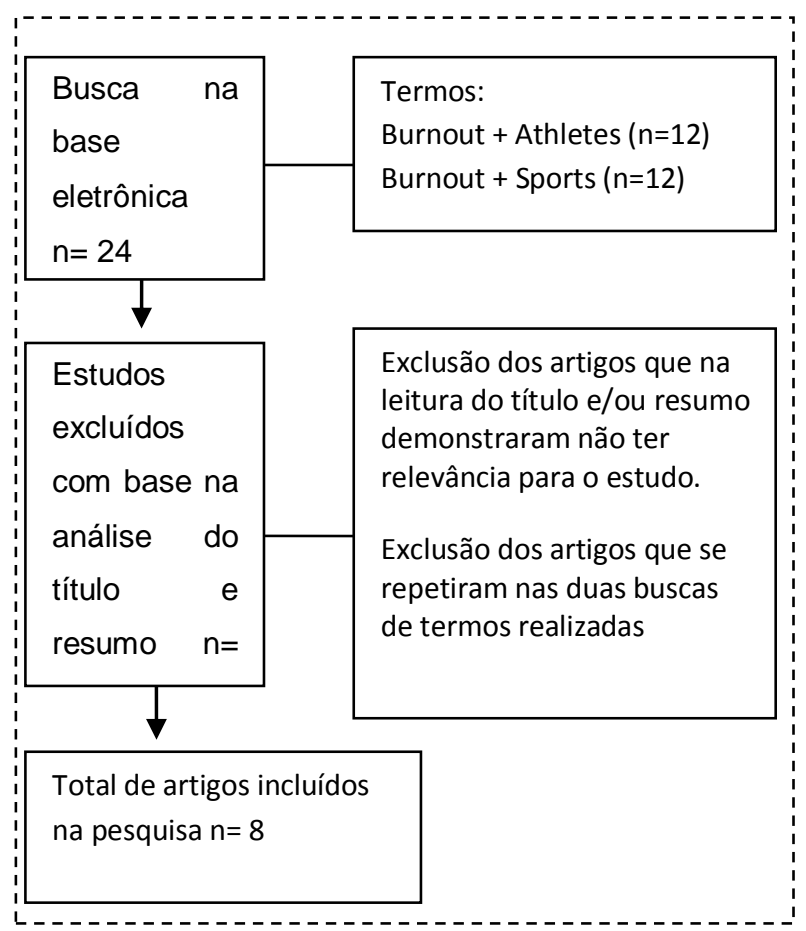

Figura 01 - Fluxo de inclusão e exclusão de estudos relacionados ao burnout no esporte publicados na basede dados ScienceDirect.

Após seleção dos artigos $(n=8)$, todos foram lidos na íntegra por dois pesquisadores. Após a leitura, os artigos foram tabulados mediante características identificadas como relevantes para a presente visão sistemática, considerando os seguintes itens: a) referência, b) revista, c) tipo de estudo (qualitativo ou quantitativo), d) número de participantes, e) amostra, f) população investigada, g) instrumento utilizado e h) tema. Havendo discordância entre os dois avaliadores na descrição das características dos estudos, um terceiro foi selecionado para parecer decisivo. 
A análise dos artigos selecionados através dos itens ou categorias produziu resultados que foram descritos num quadro geral abrangente e analisados e discutidos nos subitens "Perfeccionismo e Burnout; Sinais que Antecedem o Burnout; Burnout ao longo de uma temporada competitiva; Burnout em atletas que abandonaram o esporte; Paixão pelo Esporte e Burnout; Clima Motivacional e Burnout".

\section{RESULTADOS}

A partir das combinações de palavras "burnout e sports" e "burnout e athletes", foram encontrados 24 artigos na base de dados pesquisada. Após a exclusão dos artigos que não se enquadraram no tema, por meio da leitura do título e do resumo, e dos duplicados, restaram oito que compuseram a amostra analisada. Os artigos incluídos na pesquisa são apresentados na tabela 1 , destacando os seguintes dados: referência, revista onde foi publicado, tipo de estudo (qualitativo ou quantitativo), número de participantes, amostra e sexo, população investigada, instrumento utilizado e tema.

Quadro 01 - Análise dos artigos publicados em periódicos indexados, sobre a temática "burnout no esporte" dispostos na base de dados ScienceDirect.

\begin{tabular}{|c|c|c|c|c|c|c|c|}
\hline Referência & Revista & Tipo de estudo & $\mathbf{N}$ & $\begin{array}{l}\text { Amostra } \\
\text { Sexo }\end{array}$ & $\begin{array}{l}\text { População } \\
\text { investigada }\end{array}$ & Instrumento & Tema \\
\hline $\begin{array}{l}\text { Cresswell e } \\
\text { Eklund (2004) }\end{array}$ & $\begin{array}{c}\text { Journal of } \\
\text { Science and } \\
\text { Medicine in Sport }\end{array}$ & Quantitativo & 199 & $\begin{array}{l}\text { Atletas de } \\
\text { rugby } \\
\text { Homens }\end{array}$ & $\begin{array}{c}\text { Atletas profissionais } \\
\text { e quase } \\
\text { profissionais } \\
\text { Nova Zelândia }\end{array}$ & $A B Q$ & $\begin{array}{l}\text { Burnout e os sinais } \\
\text { que o antecedem }\end{array}$ \\
\hline $\begin{array}{l}\text { Cresswell e } \\
\text { Eklund (2006) }\end{array}$ & $\begin{array}{c}\text { Journal of } \\
\text { Science and } \\
\text { Medicine in Sport }\end{array}$ & Quantitativo & 109 & $\begin{array}{l}\text { Atletas de } \\
\text { rugby } \\
\text { * }\end{array}$ & $\begin{array}{l}\text { Atletas profissionais } \\
\text { Nova Zelândia }\end{array}$ & $\mathrm{ABQ}$ & $\begin{array}{l}\text { Variação no } \\
\text { burnout ao longo de } \\
\text { uma temporada } \\
\text { competitiva }\end{array}$ \\
\hline Hill et al. (2008) & $\begin{array}{c}\text { Psychology of } \\
\text { Sport and } \\
\text { Exercise }\end{array}$ & Quantitativo & 151 & $\begin{array}{l}\text { Atletas de } \\
\text { futebol } \\
\text { Homens }\end{array}$ & $\begin{array}{l}\text { Atletas juvenis } \\
\text { Reino Unido }\end{array}$ & $\mathrm{ABQ}$ & $\begin{array}{c}\text { Burnout e } \\
\text { perfeccionismo }\end{array}$ \\
\hline $\begin{array}{l}\text { Gustafsson et al. } \\
(2008)\end{array}$ & $\begin{array}{c}\text { Psychology of } \\
\text { Sport and } \\
\text { Exercise } \\
\text {. }\end{array}$ & $\begin{array}{c}\text { Qualitativo / } \\
\text { Quantitativo } \\
\text { Empírico }\end{array}$ & $628 / 10$ & $\begin{array}{c}\text { Atletas de } \\
\text { diferentes } \\
\text { modalidades } \\
\text { Homens e } \\
\text { Mulheres }\end{array}$ & $\begin{array}{c}\text { Atletas profissionais } \\
\text { Suíça }\end{array}$ & $\begin{array}{c}A B Q \text { e } \\
\text { entrevista }\end{array}$ & $\begin{array}{c}\text { Burnout em atletas } \\
\text { que abandonaram } \\
\text { o esporte }\end{array}$ \\
\hline
\end{tabular}




\begin{tabular}{|c|c|c|c|c|c|c|c|}
\hline Cresswell (2009) & $\begin{array}{c}\text { Journal of } \\
\text { Science and } \\
\text { Medicine in Sport }\end{array}$ & Quantitativo & 183 & $\begin{array}{l}\text { Atletas de } \\
\text { rugby } \\
\text { Homens }\end{array}$ & $\begin{array}{c}\text { Atletas profissionais } \\
\text { Inglaterra }\end{array}$ & $A B Q$ & $\begin{array}{l}\text { Burnout e os sinais } \\
\text { que o antecedem }\end{array}$ \\
\hline $\begin{array}{l}\text { Applenton, Hall e } \\
\text { Hill (2009) }\end{array}$ & $\begin{array}{l}\text { Psychology of } \\
\text { Sport and } \\
\text { Exercise }\end{array}$ & Quantitativo & 201 & $\begin{array}{c}\text { Atletas de } \\
\text { diferentes } \\
\text { modalidades } \\
\text { Homens }\end{array}$ & $\begin{array}{c}\text { Atletas juvenis de } \\
\text { elite } \\
\text { Reino Unido }\end{array}$ & $A B Q$ & $\begin{array}{c}\text { Burnout e } \\
\text { perfeccionismo }\end{array}$ \\
\hline $\begin{array}{l}\text { Smith, Gustafsson } \\
\text { e Hassmén (2010) }\end{array}$ & $\begin{array}{l}\text { Psychology of } \\
\text { Sport and } \\
\text { Exercise }\end{array}$ & Quantitativo & 206 & $\begin{array}{c}\text { Atletas de } \\
\text { diferentes } \\
\text { modalidades } \\
\text { Homens e } \\
\text { Mulheres }\end{array}$ & $\begin{array}{c}\text { Atletas juvenis de } \\
\text { elite } \\
\text { Suécia }\end{array}$ & $A B Q$ & $\begin{array}{l}\text { Burnout e clima } \\
\text { motivacional }\end{array}$ \\
\hline $\begin{array}{l}\text { Curran et al. } \\
\text { (2011) }\end{array}$ & $\begin{array}{l}\text { Psychology of } \\
\text { Sport and } \\
\text { Exercise }\end{array}$ & $\begin{array}{l}\text { Quantitativo } \\
\text { Empírico }\end{array}$ & 149 & $\begin{array}{c}\text { Atletas futebol } \\
\text { Homens }\end{array}$ & $\begin{array}{l}\text { Atletas juvenis de } \\
\text { elite } \\
\text { Reino Unido }\end{array}$ & $A B Q$ & $\begin{array}{c}\text { Paixão pelo esporte } \\
\text { e burnout }\end{array}$ \\
\hline
\end{tabular}

A primeira publicação registrada nos periódicos investigados ocorreu no ano de 2004, havendo um aumento de artigos relacionados ao burnout no esporte a partir de 2008. Entre os artigos relacionados à temática, houve predominância de pesquisas quantitativas $(n=7)$, havendo apenas uma com abordagem qualitativa. As publicações se concentraram em duas revistas, 0 Journal of Science and Medicine in Sport $(\mathrm{n}=3)$ e o Psychology of Sport and Exercise $(\mathrm{n}=5)$.

Em relação ao número de participantes, as pesquisas quantitativas variaram entre 109 e 628 sujeitos (média de 228). A pesquisa qualitativa foi realizada por meio de entrevista com 10 sujeitos. Todas as pesquisas utilizaram o Athlete Burnout Questionaire (ABQ)como instrumento para avaliar o burnout. Apenas uma pesquisa utilizou-se, além $A B Q$ para avaliar o burnout, de entrevista.

As populações investigadas nos estudos foram de atletas de elite juvenis, atletas quase profissionais e atletas profissionais de diferentes países (Nova Zelândia, Reino Unido, Suíça, Inglaterra e Suécia). Em relação à amostra, são comuns atletas tanto de modalidades individuais quanto coletivas. As modalidades investigadas foram: rugby, futebol de campo, tênis de mesa, esqui crosscountry, natação, vôlei, boliche, basquetebol, triatlo, biatlo, atletismo e cricket. Entretanto, não foi possível caracterizar todas as modalidades, pois 
alguns estudos não especificaram quais eram. Em relação ao sexo, cinco estudos investigaram apenas homens, dois investigaram homens e mulheres, e um estudo não especificou o sexo dos participantes.

Em relação aos temas dos artigos incluídos na pesquisa, Hill et al. (2008) e Applenton, Hall e Hill (2009) verificaram a relação entre o burnout e o perfeccionismo; Cresswell e Eklund (2004) e Cresswell (2009) estudaram o burnout e os sinais que o antecedem; Gustafsson et al.(2008) investigaram como burnout é vivenciado, seus antecedentes e consequências percebidas por atletas que abandonaram o esporte; Cresswell e Eklund (2006) investigaram as variações nos níveis de burnout e suas dimensões ao longo de uma temporada competitiva; Curran et al. (2011) relacionou a paixão pelo esporte e o burnout; e Smith, Gustafsson e Hassmén (2010) verificaram a relação entre o clima motivacional e o burnout.

\section{DISCUSSÃO}

O burnout é uma temática que teve suas primeiras aparições na literatura científica há quase 40 anos, sendo que os primeiros estudos são encontrados na área da Psicologia, especialmente na Psicologia Organizacional. Os estudos sobre burnout no meio esportivo são relativamente recentes, como apontaram Goodger et al.(2007) em revisão sistemática sobre o tema. Entre os 61 artigos encontrados em sua busca, 27 tinham como objeto principal o de estudo do burnout em atletas.

De acordo com Raedeke e Smith (2001), os estudos sobre o burnout em atletas têm sido dificultados pela falta de instrumentos validados. O primeiro instrumento desenvolvido exclusivamente para a mensuração do burnout esportivo foi o Eades Athlete Burnout Inventory (RAEDEKE; SMITH, 2001). Uma década depois, Raedeke e Smith (2001) criaram o Athlete Burnout Questionaire ( $A B Q$ ), que apesar de ter sido baseado inicialmente no Eades Athlete Burnout Inventory (possuindo inclusive questões iguais ao primeiro, apresenta uma relação mais direta com as dimensões propostas por Maslack e Jackson (1981) (RAEDEKE; SMITH, 2001).

$\mathrm{Na}$ busca realizada no presente estudo, todas as pesquisas utilizaram como instrumento de coleta de dados o ABQ. Apenas uma pesquisa utilizou, 
além do $A B Q$, uma entrevista para aprofundar os questionamentos sobre o burnout. O ABQ tornou-se muito popular nos últimos anos (GOODGER et al., 2007) e parece ser o questionário mais indicado para se mensurar o burnout em atletas. Além disso, o $A B Q$ possui versões validadas em diversos países, inclusive no Brasil com o Questionário de Burnout para Atletas (QBA), validado por Pires Brandão e Silva (2006).

$O A B Q$ foi inicialmente composto por 15 itens divididos em três subescalas de 5 afirmações (RAEDEKE; SMITH, 2001). Cada subescala se refere a um construto relacionado à manifestação de burnout em atletas. As dimensões são: (a) baixo senso de realização esportiva (ex.: parece que não importa o que eu faça, eu não desempenho tão bem quanto deveria, (b) desvalorização esportiva (ex.: eu tenho sentimentos negativos em relação ao esporte), e (c) exaustão física e emocional (ex.: eu me sinto exausto pelas demandas físicas e mentais do meu esporte). Para cada afirmação o respondente se posiciona dentro de uma escala do tipo Likert que varia de 1 (quase nunca) a 5 (quase sempre) (RAEDEKE; SMITH, 2001).

$\mathrm{Na}$ Psicologia do Esporte e do Exercício os métodos quantitativos são historicamente mais utilizados. Biddle (1997) analisou os artigos $(n=529)$ publicados entre 1985 e 1994 nas duas principais revistas da Psicologia do Esporte nesse período (Journal of Sport Psychology e Journal of Sport and Exercise Psychology). Menos de 6\% dos artigos utilizavam métodos qualitativos. Um editorial do Psysicology of Sport and Exercise de 2000 também apontava a necessidade de mais estudos qualitativos (EDITORIAL 2000). Verifica-se que na área do burnout esportivo a tendência é a mesma, pois $87 \%$ dos estudos revisados lançaram mão exclusivamente de métodos qualitativos ( $n=7)$.

A seguir são apresentados os estudos revisados, separando-os por tema.

\section{Perfeccionismo e Burnout}

Segundo Jackson e Roderick, citados por Hill et al. (2008), a pressão para atingir metas e objetivos é uma condição importante para o desenvolvimento do burnout. Baseado nisso, Hill et al.(2008) investigaram a relação entre o perfeccionismo (self-oriented e soccialy prescribed) e o burnout, 
e como a auto-aceitação media essa possível relação. No segundo estudo, Applenton, Hall e Hill (2009) investigaram a hipótese de que a satisfação do atleta perante os seus objetivos atingidos e as suas orientações para 0 objetivopoderiam mediar associações entre o burnout e o perfeccionismo selforiented e soccialy prescribed.

O perfeccionismo self-oriented é caracterizado pela busca de padrões excessivamente elevados de desempenho, acompanhada por uma autocrítica severa e potencialmente debilitante. Já o perfeccionismo soccialy prescribed envolve a percepção de que pessoas significantes para o atleta impõem padrões e metas a ele, e que estas são avaliadas rigorosamente. Na avaliação do atleta, cumprindo essas metas terá a aprovação significativa das pessoas (HILL et al., 2008).

Ambos os estudos encontraram resultados semelhantes. Applenton, Hall e Hill (2009) e Hill et al. (2008) verificaram uma relação positiva entre o perfeccionismo soccialy prescribed e as três dimensões do burnout (exaustão física e emocional, desvalorização esportiva, baixo senso de realização esportiva), enquanto o perfeccionismo self-oriented esteve associado inversamente com as três dimensões. Dessa maneira, a pressão de técnicos, família e patrocínios, por exemplo, poderiam ser um facilitador para 0 desenvolvimento do burnout. Quanto a auto-aceitação, Hill et al. (2008) verificaram que quando ela é negativa ambas as formas de perfeccionismo podem ser antecedentes de burnout, pois a busca pela autoaceitação pode ser um processo psicológico crítico para o desenvolvimento da síndrome.

\section{Sinais que Antecedem o Burnout}

Cresswell e Eklund (2003) propuseram um guia da síndrome de burnout, no qual foram propostos sinais para indicar se o atleta poderia estar em risco de burnout. Entretanto, para Cresswell (2009) os sintomas propostos poderiam significar na verdade que 0 atleta estaria experimentando/vivenciando 0 burnout. Para verificar essa hipótese, Cresswell e Eklund (2004) analisaram as possíveis associações entre esses sinais e as dimensões do burnout, enquanto Cresswell (2009) avaliou se esses mesmos sinais, mensurados no meio de uma temporada competitiva, teriam associações com o burnout mensurado ao final da mesma temporada. 
Tanto no estudo de Cresswell e Eklund (2004), quanto no de Cresswell e Eklund (2009), os resultados indicaram associações significativas entre os sinais que antecederiam o burnout e a própria síndrome. Ambos os estudos verificaram associações positivas entre o burnout e problemas relacionados ao esporte em si e problemas financeiros. Cresswell e Eklund (2009) encontraram associações negativas entreas percepções de apoio social e o burnout, de modo que quem recebia mais apoio de seus companheiros de esporte apresentava menor burnout. Entretanto, no estudo de Cresswell e Eklund (2004), além da percepção de adequado suporte social, a percepção de competência e autonomia foram negativamente correlacionadas com as principais características do burnout.

\section{Burnout ao longo de uma temporada competitiva}

O estudo de Cresswell e Eklund (2006) comparou os escores de burnout ao longo de uma temporada competitiva de 30 semanas, buscando analisar as possíveis variações no burnout e suas dimensões. O estudo aconteceu em três momentos, período pré-competição, durante a competição e pós-competição, analisando fatores como número de lesões sofridas, nível competitivo, posição do jogador e anos de experiência competindo na liga em questão. Pesquisas

anteriores já haviam estudado essas variações, porém dentro de uma abordagem unidimensional (LAl e WIGGINS, 2003). Os achados do estudo de Cresswell e Eklund (2006) evidenciam que o burnout em atletas flutua em relação às mudanças situacionais e demandas do ambiente. Os sentimentos de baixo senso de realização esportiva aumentaram da fase pré-competitiva para a competitiva. Mudanças na exaustão física e emocional ao longo da temporada foram associadas com as posições dos jogadores, sendo que algumas posições sofrem mais em relação a essa dimensão. O burnout também esteve associado a lesões, não ser selecionado para jogar e à menor experiência na modalidade.

\section{Burnout em atletas que abandonaram o esporte}

Gustafsson et al. (2008) tiveram como objetivo investigar o burnout, seus antecedentes e as conseqüências percebidas por atletas que abandonaram o esporte. A partir de uma amostra de 628 sujeitos, os que obtiveram os maiores 
escores de burnout participaram de um segundo estudo baseado em uma entrevista retrospectiva. Uma das principais contribuições do estudo foi reforçar o aspecto multidimensional da síndrome proposto por Raedeke (1997). Estressores como "baixa recuperação", "muito esporte" e "altas expectativas" foram relatados pelos entrevistados como sendo causas do burnout. As diferentes demandas como demandas do esporte, da escola, e de sua vida social são também agravantes do problema, e segundo o autor contribuem para um estado de sobrecarga total.

\section{Paixão pelo Esporte e Burnout}

A paixão é definida como uma inclinação forte para alguma atividade que a pessoa desfruta, acha importante, investe tempo e energia nessa atividade (VALLERAND et al., 2003). Apesar de a paixão estimular a participação voluntária na atividade, ela tem potencial para sustentar resultados tanto positivos como negativos. Vallerand et al. (2003) sugerem que a maneira como os indivíduos internalizam a atividade (motivações mais internas ou externas) determina qual tipo de paixão irá se desenvolver. A internalização mais autônoma resulta em uma paixão harmoniosa, enquanto uma internalização mais controlada tende a desenvolver uma paixão obsessiva (Vallerand, 2008).

O estudo de Curran et al. (2011) examinou as possíveis associações entre os dois tipos de paixão, harmoniosa e obsessiva, e o burnout. Os resultados do estudo demonstraram associação negativa entre o burnout e a paixão harmoniosa. A partir desses resultados, os autores sugerem que a paixão harmoniosa pode oferecer algum tipo de proteção contra o burnout em função de motivações mais autodeterminadas. A hipótese de que a paixão obsessiva seria positivamente associada ao burnout não foi confirmada.

\section{Clima Motivacional e Burnout}

Estudos baseados nas teorias da motivação mostram que o clima motivacional (LEMYRE, HALL e ROBERTS, 2008; REINBOTH e DUDA, 2004) e a atmosfera de conflito entre os jogadores (LEMYRE, HALL e ROBERTS, 2008; REINBOTH e DUDA, 2004) estão associados ao burnout. Nessa perspectiva, o estudo de Smith, Gustafsson e Hassmén (2010)teve como 
objetivo investigar de que forma o clima motivacional entre os atletas e seus companheiros de esporte estavam associadas ao burnout. O clima motivacional percebido pode ser diferenciado em task involving ou ego involving. Quanto ao task involving, é possível se segmentar em improvement (ex.: ajudar o outro a melhorar), relatedness support (ex.: fazer os companheiros de time se sentirem valiosos) e effort (ex.: encorajar os companheiros de time a tentar o seu melhor). Em relação ao ego involving, o mesmo pode ser dividido em intra-teamcompetitionand ability (ex.: encorajar os companheiros de esporte a um ser melhor que o outro) e intra-team conflict (ex.: fazer comentários negativos que colocam seus companheiros pra baixo). As variáveis de controle do estudo foram as horas de treinamento e o estresse percebido.

Os resultados apontaram uma correlação positiva entre conflitos intrateam conflict e o burnout nos esportes individuais. As participantes mulheres em relação aos homens apresentaram escores mais altos nas variáveis de controle e no componente effort e escores mais baixos em todos os componentes do ego involving. Não foram encontradas associações entre intrateam competition e ability e o burnout,diferente do que foi hipotetizado. Finalmente, o clima motivacional predisse melhor o burnout do que as variáveis de controle, demonstrando que a motivação é um fator fundamental no desenvolvimento do burnout.

\section{CONSIDERAÇÕES FINAIS}

A presente pesquisa analisou as publicações sobre burnout no esporte na base de dados Sciencedirect, um dos principais meios de divulgação científica mundial. Trata-se de um tema pouco publicado na literatura internacional, especialmente com relação à realidade brasileira.

A análise demonstrou que as publicações sobre o burnout vêm sendo realizadas com atletas de diferentes modalidades, tanto coletivas como individuais, em diferentes países. Encontrou-se ainda uma preferência por estudos quantitativos e observou-se que o principal instrumento de coleta de dados é o Athlete Burnout Questionaire (ABQ). As variáveis perfeccionismo, 
sinais que antecedem o burnout, clima motivacional e paixão estiveram associadas ao burnout.

O resultado dos estudos apontaram associações positivas entre 0 perfeccionismo soccialy prescribed e o burnout. Foram encontradas também associações negativas entre o burnout e o perfeccionismo self-oriented e a paixão harmoniosa. Os sinais que antecedem o burnout que foram propostos tiveram de fato associação com a síndrome. Foi reforçado o conceito de que o burnout tem origens multidimensionais e que o mesmo sofre influência do ambiente social através do clima motivacional.

\section{REFERÊNCIAS}

APPLETON, P.R; HALL, H.K; HILL, A.P. Relations between multidimensional perfectionism and burnout in junior-elite male athletes. Psychology of Sport and Exercise, v.10, n.4, p.457-465, 2009.

BIDDLE, S.J. Current trends in Sport and exercise psychology research. The Psychologist: Bulletin of the british psychological society, v.10, n.2, p.6369, 1997.

CRESSWELL, S.L. Possible early signs of athlete burnout: A prospective study. Journal of Science and Medicine in Sport, v.12, n.3, p.393-398, 2009.

CRESSWELL, S.L.; EKLUND R.C. The athlete burnout syndrome: A practitioner's guide. New Zealand Journal of Sports Medicine, v.31, p. 4-9, 2003.

CRESSWELL, S.L; EKLUND, R.C. Changes in athlete burnout over a thirtyweek "rugby year". Medicine and Science in Sports and Exercise, v.9, p.125134, 2006.

CRESSWELL, S.L; EKLUND, R.C. The athlete burnout syndrome: possible early signs. Journal ofScience and Medicine in Sports and Exercise, v.7, n. 4, p.481-487, 2004. 
CURRAN, T; APPLENTON, P.R; HILL, A.P; HALL, H.K. Passion and burnout in elite junior soccer players: the mediating role of self-determined motivation. Psychology of Sport and Exercise, v.12, n.6, p.655-661, 2011.

EDITORIAL. Psychology of Sport and Exercise - present and future. Psychology of Sport and Exercise, v.1, p.1-5, 2000.

FERREIRA NETO, A; NASCIMENTO, A.C.S. Periódicos científicos da Educação Física: Proposta de Avaliação. Revista Movimento, v.8, n.2, p. 3549, 2002.

FLIPPIN, R. Burning out. The Runner, v.32, p.77-83, 1981.

FREUDENBERGER, H.J. Staff burnout. Journal of Social Issues, v.30, n.1, p.159-165, 1974.

GOODGER, K; GORELY, T; LAVALLEE, D; HARWOOD, C. Burnout in Sport: A Systematic Review. The Sport Psychologist, v.21, n.2, p. 127-151, 2007.

GUSTAFSSON, H; HASSMÉN, P; KENTTA, G; JOHANSSON, M. A quantitative analysis of burnout in elite Swedish athletes. Psychology of Sport and Exercise, v.9, n.6, p.800-816, 2008.

HILL, A.P; HALL, H.K; APPLETON, P.R; KOZUB, S.A. Perfectionism and burnout in junior elite soccer players: The mediating influence of unconditional self-acceptance. Psychology of Sport and Exercise, v.9, n.5, p.630-644, 2008.

JSSM. JOURNAL OF SPORT SCIENCE AND MEDICINE. Revista eletrônica. Disponível em < http://www.jssm.org/>. Acesso em : 08/02/2012.

LAI, C.; WIGGINS, M. S. Burnout perceptions over time in NCAA division I soccer players. International Sports Journal, v.7, n.2, p. 120-127, 2003. 
LEMYRE, P.N.; HALL, H. K.; ROBERTS, G. C. A social cognitive approach to burnout in elite athletes. Scandinavian Journal of Medicine \& Science in Sports, v.18, n.2, 221-234, 2008.

MASLACH, C; JACKSON, S.E. The measurement of experienced burnout. Journal of occupational behaviour, v.2, n.2, p.99-113, 1981.

PIRES, D.A; BRANDÃO, M.R.F; MACHADO, A.A. A síndrome de Burnout no esporte. Revista Motriz, Rio Claro, v.11, n.3, p.147-153, 2005.

PIRES, D.A; BRANDÃO, M.R.F; SILVA, C.B. Validação do Questionário de Burnout para Atleta. Revista da Educação Física/UEM, v.17, n.1, p.2736, 2006.

PSE. PSYCHOLOGY OF SPORT AND EXERCISE. Revista eletrônica. Disponível em < http://www.journals.elsevier.com/psychology-of-sport-andexercise/> Acesso em : 08/02/2012.

RADAEKE, T. Is athlete burnout more than Just stress? A sport commitment perspective. Journal of Sport and Exercise Psychology. v.19, n.4, p.396417, 1997.

RADAEKE, T; LUNNEY, K; VENABLES, K. Understanding athlete burnout: coach perspectives. Journal of Sports Behaviors. v.25, n. 2, p.181-206, 2002.

RADAEKE, T; SMITH, A. Development and preliminary validation of an athlete Burnout measure. Journal of Sport and Exercise Psychology. v.23, n.4, p.281-306, 2001.

REINBOTH, M.; DUDA, J. L. The motivational climate, perceived ability, and athletes' psychological and physical well-being. The Sport Psychologist, v.18, n.3, p. 237-251, 2004. 
SCIENCE DIRECT : base de dados. Disponível em $<$ http://www.sciencedirect.com>. Acesso em: 08/02/2012.

SMITH, A.L; GUSTAFSSON, H; HAMSSÉN, P. Peer motivational climate and burnout perceptions of adolescent athletes. Psychology of Sport and Exercise, v.11, n.6, p.453-460, 2010.

SMITH, R.E. Toward a cognitive-affective model of athlete burnout. Journal of Sport Psychology. v.8, n.1, p. 36-50, 1986.

VALLERAND, R. J., MAGEAU, G. A., ELLIOT, A. J., DUMAIS, A., DEMERS, M.-A., ROUSSEAU, F. Passion and performance attainment in sport. Psychology of Sport and Exercise, v.9, n.3, p.373-392, 2008.

VALLERAND, R. J.; BLANCHARD, C.; MAGEAU, G. A.; KOESTNER, R.; RATELLE, C. F.; LÉONARD, M.; GAGNÉ, M; MARSOLAIS,J. Les passions de l'Âme: On obsessive and harmonious passion. Journal of Personality and Social Psychology, v.85, n.4 p.756-767, 2003. 


\title{
BURNOUT IN SPORT: SISTEMATIC REVIEW IN ScienceDirect DATABASE
}

\begin{abstract}
This study had the purpose of analyzing the scientific production related to burnout in sports from articles published in the last decade (2002-2011) in electronic database ScienceDirect, since it is relevant, recognized, representative and comprehensive. From the combinations of words "sports burnout" and "burnout and Athletes", eight articles were selected for the study. The items investigated were: journal, study type, number of participants, sample, studied population, instruments used, subject and results .Publications concentrated on the Journal of Science and Medicine in Sport and Exercise Psychology of Sport and quantitative surveys predominated $(n=7)$ with an average of 228 participants. The populations studied were young elite athletes, almost professional athletes and professional athletes from different countries, Europe and Oceania. All studies used the Athlete Burnout Questionnaire (ABQ) as a tool for data collection. On the subject there were identified and presented results of articles about perfectionism and burnout $(n=2)$, signs that precede burnout $(n=2)$, burnout, its antecedents and consequences for athletes who have left the sport $(n=1)$ variations in burnout levels throughout a competitive season $(n=1)$, passion for the sport and burnout $(n=1)$ and motivational environment and burnout $(n=1)$.
\end{abstract}

Key words: Burnout, sport, athlete, psychology.

\section{BURNOUT EN EL DEPORTE: REVISIÓN SISTEMÁTICA EN LA BASES ScienceDirect}

\section{RESUMEN}

Este estudio tuvo como objetivo analizar la producción científica relacionada con el burnout en los deportes partiendo de artículos publicados en la última década (2002-20011) en bases de datos electrónicos de ScienceDirect, ya que esta es relevante, reconocida, representativa y global. A partir de las combinaciones de las palabras "burnout y deporte" y "burnout y atletas", ocho artículos fueron seleccionados para el estudio. Los elementos investigados fueron: periódico, tipo de estudio, número de participantes, amuestra, población estudiada, instrumentos utilizados, temáticas y los resultados. Las publicaciones están centradas en los periódicos Journal of Science and Medicine is Sport e Psychology of Sport and Exercise, predominaron los estudios cuantitativos $(n=7)$ con una media de 228 participantes. Las poblaciones estudiadas fueron los jóvens atletas de élite, los atletas case profesionales y atletas profesionales de diferentes países, Europeos y de la Oceanía. Todos los estudios utilizaron el Athlete Burnout Questionaire (ABQ) como una herramienta para la recogida de datos. Con relación al tema se identificaron y presentaron los resultados de artículos sobre burnout y el perfeccionismo $(n=2)$, señales que preceden el burnout $(n=2)$, burnout, sus antecedentes y conseceuncias pera los atletas que han abandonado el deporte $(n=1)$ variaciones en los niveles de burnout en toda una temporada de competición $(n=1)$, la pasión por el deporte y el burnout $(n=1)$ y clima motivacional y el burnout $(n=1)$.

Palabras clave: Burnout. Deportes. Atleta. Psicologia.

Endereço para correspondencia: alexandro.andrade@udes.br

\footnotetext{
* Doutora em Ciências do Movimento Humano (PPGCMH/UFRGS) e docente do Centro de Educação Física e Desportos (CEFD) da Universidade Federal de Santa Maria (UFSM) e integrante da Equipe Editorial da Revista Kinesis.

*** Acadêmica(bolsista) do Curso de Educação Física(Licenciatura) do CEFD/UFSM e integrante da Equipe Editorial da Revista Kinesis.

${ }^{* * * *}$ Técnico administrativo do CEFD/UFSM e integrante da Equipe Editorial da Revista Kinesis.
} 